\title{
BUCHKRITIK
}

\section{Auf der Suche nach dem Blick von Nirgendwo}

\author{
Von ANTON LEIST (Zürich)
}

GOTTFRIED SEEBASS: HANDLUNG UND FREIHEIT. Philosophische Aufsätze. Mohr Siebeck Verlag, Tübingen 2006, 411 S. (= a).

GOTTFRIED SEEBASS: WILLENSFREIHEIT UND DETERMINISMUS. Band 1: Die Bedeutung des Willensfreiheitsproblems. Akademie Verlag, Berlin 2007, 236 S. (= b).

Stellen wir uns vor, die Welt wäre vollständig determiniert. Wären wir in unserem Wollen und Handeln dennoch frei? Oder umgekehrt, stellen wir uns vor, unser Wollen und Handeln wäre frei. Wäre dann die Welt indeterminiert? Unterstellt man einen vollständigen Gegensatz von Freiheit und Determinismus, fallen diese Fragen zusammen. Das Verhältnis von Freiheit und Determinismus ist aber so unklar wie diese Phänomene selbst, sodass sich die eine Frage unabhängig von der anderen in den Vordergrund schieben kann. In der Regel dominiert die erste Frage, die sich auf die Interpretation von Freiheit angesichts des möglichen Determinismus konzentriert. Dieses vorrangige Interesse am Freiheitsverständnis scheint sich dadurch zu ergeben, dass uns die vermeintlichen Freiheitsaspekte unseres Handelns leichter zugänglich sind als die Bedeutungen des Determinismus; es kann aber auch bedeuten, dass unsere Freiheitsbedürfnisse unausweichlich den Rahmen festlegen, innerhalb dessen der Konflikt zwischen Determinismus und Freiheit entschieden werden muss.

Seebaß' gelehrte und umsichtige Diskussion der Willensfreiheit folgt der üblichen Strategie, die Herausforderung des Determinismus mittels einer Analyse von Freiheit zu prüfen. Die meisten einschlägigen Artikel in Handlung und Freiheit verfolgen den Zweck, angesichts des möglichen Determinismus die Bedeutung einer „ontologischen Offenheit“ nachzuweisen. ${ }^{1}$ Angesichts dieses Interesses an ontologischer Offenheit, häufig auch gleichgesetzt mit „Indeterminiertheit“, wäre eine ausführlichere Beschäftigung mit dem Determinismus angebracht, als beide Bände sie enthalten. ${ }^{2}$ Unter ,Determinismus ' wird von Seebaß wie von vielen anderen der einfache Gedanke verstanden, dass die Zukunft der Welt in allen ihren Erscheinungen festgelegt sei (a, 132), und gegen diese Vorstellung richtet sich die Forderung

1 Auf Grund der herausragenden Bedeutung des Problems der Willensfreiheit in beiden Büchern werde ich mich auf die einschlägigen (vier von neun) Kapitel in (a) beschränken, auf die der Autor in (b) als auf Vorarbeiten Bezug nimmt (b, 14 f.). (b) liegt als erster Band einer dreibändig geplanten Studie vor und lässt ein spezielles Profil des zentralen Problems noch kaum erkennen. Die nachfolgenden Bemerkungen beziehen sich deshalb schwergewichtig auf (a).

2 (a), 131-136. (b) stellt sie für den zweiten geplanten Band in Aussicht. 
der, ontologischen Offenheit'. Vergleiche mit deterministischen Maschinen wie Schachcomputern (mit oder ohne Zufallselementen) zeigen jedoch, dass sich kaum bestreiten lässt, dass Deep Blue der Urheber genialer Züge ist, und dass die Vorstellung unserer eigenen vollständigen Determiniertheit als etwas komplexerer biologischer Maschinen deshalb nicht unbedingt unsere freie Urheberschaft von Handlungen erschüttern muss. ${ }^{3}$ Warum sollten wir nicht deterministisch frei sein, wenn Deep Blue deterministisch genial sein kann?

\section{Die ontologische Drohung des Determinismus}

Die generelle argumentative Bewegung in den einschlägigen Artikeln von Handlung und Freiheit lässt sich damit zusammenfassen, dass Seebaß den verschiedenen Ansätzen zu einer ,konditionalen Analyse“ von Willensfreiheit die Drohung des Determinismus entgegenhält. Etwas anders könnte man auch sagen, dass Seebaß die ,ontologische Intuition' verbalisiert, wonach das menschliche Leben in einem deterministischen Universum gegenüber diesem Universum nicht frei sein kann. Dies ist eine vereinfachende und interpretierende Wiedergabe, weil die einzelnen Artikel eine im Detail häufig überkomplexe Vielfalt von Gedanken versammeln und weil sich Seebaß selbst nicht so äußert, dass er dieser ontologischen Intuition anhinge und einen Gegensatz von Willensfreiheit und Determinismus unterstellte. In seiner Vorgehensweise vermittelt er vielmehr den Eindruck der völligen Neutralität gegenüber kontroversen metaphysischen Annahmen. Ein Blick auf den Gedankengang des Artikels „Freiheit und Determinismus" in Handlung und Freiheit kann das stellvertretend für alle anderen verdeutlichen.

Zunächst schildert Seebaß die durch die Quantenphysik unsicher gewordenen Evidenzen für einen physikalischen Determinismus und betont, inwieweit der heute obsolet gewordene Glaube an den ,Satz vom Grund' die Vorstellung vom Determinismus beeinflusst hat. Ist der Determinismus als rein theoretisches und auf Grund seiner Allaussage vermutlich nie zu entscheidendes Konstrukt keine Bedrohung, so doch der „Fatalismus“, den Seebaß als die praktische Furcht vor dem Determinismus viel ernster nimmt, obwohl letztlich unklar bleibt, wieso. Angesichts des Fatalismus schwenkt er auf die Alternative ein, dass entweder eine nicht-ontologische oder eine ontologische Analyse des ,praktischen Könnens“ angezeigt sei (a, 141). Die nicht-ontologische Analyse identifiziert er mit der kompatibilistischen Tradition. Nicht-ontologisch verstehen sich Kompatibilisten deshalb, weil sie meinen, allein mit einer Analyse des Wollens dem Phänomen Freiheit hinreichend gerecht werden zu können. Das allgemeine Muster dieser Analyse ist die von der Handlungs- auf die Willensfreiheit übertragene ,konditionale Analyse‘. Seebaß schildert verschiedene Varianten dieser Analyse, die er sukzessive für untauglich erklärt, und gelangt damit zu dem Ergebnis, dass tatsächlich eine ontologische Erklärung des praktischen Könnens nötig sei. „Ohne die Annahme partieller ontologischer Offenheit" könnten wir an unserem gewöhnlichen praktischen Selbstverständnis „nicht mehr festhalten“ (a, 165). Der damit wichtige Begriff der ontologischen Offenheit wird nicht erörtert, man muss sich ihn wohl als einen nicht-determinierten ,Spalt' in der ,Welt‘ vorstellen.

Vom Fatalismus ist Seebaß offensichtlich stärker beeindruckt, als er durch Argumente zu belegen vermag. Seine Kritik an Pothasts Hinweis, die wissensmäßige Verborgenheit unserer Zukunft in einem determinierten Leben mache unseren praktischen Standpunkt nicht überflüssig (a, 334, Fn. 20), reduziert sich im Grunde auf den Vorwurf, Pothast nehme den

3 S. C. Taylor u. D. Dennett, Who's Afraid of Determinism? Rethinking Causes and Possiblitities, in: R. Kane (Hg.), The Oxford Handbook of Free Will, Oxford 2002, 257-277. 
Determinismus nicht ernst genug. Aristoteles' vermeintlichen Fatalismus in De Interpretatione scheint Seebaß deshalb zu teilen, weil er seine Korrespondenztheorie der Wahrheit teilt (a, 131) und über sie die Determinismusproblematik ähnlich wie Aristoteles einführt. Für einen Wahrheitsminimalisten fallen solche Gründe weg. Die vom Determinismus ausgehende Drohung ist offensichtlich nur für denjenigen fühlbar, der sich auf den Standpunkt des determinierten Universums selbst zu stellen vermag.

Wie sieht die Kritik der konditionalen Analyse nun genauer aus? Seebaß unterscheidet im genannten Artikel - und analog, teilweise ausführlicher, in den anderen - vor allem drei Varianten. Die kontrafaktische konditionale Analyse sieht Freiheit dann gegeben, wenn der Akteur entsprechend handeln könnte, würde er in einer Situation auch entsprechend wollen (a, 155-159, 162 f., 174-187, 221). Die höherstufige Analyse sieht die Konstituentien der Freiheit im reflexiven Überlegen und Wollen (a, 122-125, 159-164, 223). Die Wesensanalyse versucht für Menschen wesensgemäße Möglichkeitsspielräume anhand geeigneter Kriterien zu fixieren, um darauf hin Eingriffe in diese Spielräume als unfrei auszuweisen (a, 148-153, 211, 225-238, 251 f.). Die dritte Variante ist diejenige, die Seebaß am ausführlichsten erörtert, weil sie seiner allgemeinen Vorstellung von Freiheit (a, 209, 249-251) am ehesten entspricht. Diese allgemeine Vorstellung ist die der „Hindernisfreiheit“ (a, 212-215). ${ }^{4}$ Sie auf die Willensfreiheit restlos zu übertragen, liegt, wie mir scheint, wiederum nur von einem ontologischen Hintergrund her nahe.

Die kontrafaktische Analyse wird kaum mehr vertreten, weil klar ist, dass sie die möglicherweise einschränkenden Umstände des Entstehens eines Wollens ausklammert und damit mögliche Ursachen der Unfreiheit ignoriert. Formulierungen hingegen, die soweit hypothetisch werden, dass sie von den konkreten Wollensfähigkeiten eines Akteurs abstrahieren, wären kein Beleg mehr für dessen tatsächliche Willensfreiheit (a, 157 f., 177 f., 222). Seebaß kritisiert die konditional-kontrafaktische Analyse ausführlicher auch mit dem Nachweis, dass assertorische Konditionalsätze das modale „können“ nicht wiederzugeben vermögen, das für unser Verständnis des ,freien Könnens' nötig sei (a, 179-187). Dieser Nachweis gipfelt freilich in der meines Erachtens zu weit gehenden Vermutung, dass keinerlei wollensinterne Rekonstruktionen die Modalität des praktischen Könnens wiederzugeben vermögen und man an diesem Punkt auf die ontologische Offenheit verwiesen ist (a, 189). Die von H. Frankfurt ausgehende höherstufige Wollensanalyse kritisiert Seebaß mit dem geläufigen Regresseinwand (a, 215, 223), was Frankfurts Vorschlag deshalb nicht gerecht wird, weil er die Determinismusfrage mithilfe eines berühmt gewordenen Gedankenexperiments explizit auszuklammern versucht hat. (Ich komme auf dieses Experiment im nächsten Abschnitt zurück.)

Die Wesensanalyse wird als Präzisierungsversuch des für Akteure wesentlichen „Möglichkeitsspielraums“" verstanden, der, sofern uneingeschränkt, Freiheit garantiert (a, 148). Gegen diesen Versuch führt Seebaß zwei Einwände an. Erstens ist er der Meinung, dass die gesuchten Wesensstandards dem individuellen Können nicht gerecht werden und auch im Allgemeinen nichtssagend bleiben müssen (a, 161). Und zweitens erhebt er den Einwand, dass jede Analyse des praktischen Könnens mittels einer „wenn ... dann“-Konstruktion deshalb verfehlt sei, weil sie mit den angegebenen Bedingungen nicht begründet, warum das Erfülltsein der Bedingungen freiheitsrelevant sei (a, 163 f.). Im Hintergrund dieses zweiten

4 Die allgemeine Idee von Freiheit als Hindernisfreiheit wird im letzten Artikel von (a) am ausführlichsten erläutert, mit dem man die Lektüre des Bandes am besten beginnt. Der wichtigste, die anderen Bemerkungen zur Willensfreiheit weitgehend zusammenfassende Artikel ist der vorletzte. Dennoch lässt sich an dem von mir zuerst genannten Artikel Seebaß' ontologische Vorgehensweise bereits hinreichend studieren. 
Einwands taucht die Unterstellung auf - von der auch die Metaphorik des Möglichkeitsspielraums gesteuert ist -, dass nur eine externe, unabhängig von der Handlungsstruktur gegebene Bedingung das praktische Können ermöglicht. Und tendenziell leitet diese Annahme auch den ersten Einwand und die Rede von der, Wesensanalyse'. Ohne dies selbst zu rechtfertigen, hat Seebaß ein ontologisches Szenario der Willensfreiheit aufgebaut, in das die konditionale Analyse nicht nur nicht passt, sondern das sie vielmehr gerade vermeiden will. Seine Kritik ist dieser Analyse gegenüber deshalb unfair und seine Einwände sind zirkulär

\section{Praktische oder ontologische Offenheit?}

Seebaß gerät in sein ontologisch ausgelegtes Diskussionsszenario nicht nur, weil er mit dem Fatalismus liebäugelt, sondern auch über die Annahme, dass Freiheit einzig als ,Hindernisfreiheit" verstanden werden kann (a, 250). Da wir ,Hindernisse' zunächst nur räumlich zu denken vermögen, legt die Willens- als Hindernisfreiheit schnell einen ,Raum` nahe, in dem man nur dann frei wäre, wenn die ,Welt' , ontologisch offen ' ist, vergleichbar einer Gefängniszelle, die nur dann für den Gefangenen offen ist, wenn er ungehindert durch die Tür in die Freiheit kann. Ein Unterschied zwischen Handlungs- und Willensfreiheit besteht jedoch gerade darin, dass beim Handelnden die räumliche Vorstellung des Innen und Außen auf Grund der räumlichen Existenz des Akteurs ein Stück weit sinnvoll sein mag, wie etwa im Fall des Gefangenen. Für alle in die psychische Struktur eingewanderten Zwänge und im Fall des als frei festzulegenden Wollens versagt die räumliche Metaphorik jedoch, die Rede von einer Willens- als Hindernisfreiheit müsste von ihren räumlichen Assoziationen entsprechend gereinigt werden. Gegen das nahe liegende Argument, dass dann nicht mehr von Freiheit die Rede sei, gilt hingegen, dass die räumliche Vorstellung bereits in Fällen wie dem des Gefangenen nicht ausschließlich zutrifft. Wichtig auch am Gefangenen ist ja sein Wollen, und das kann - anders als die Bewegung seines Körpers - durch die geschlossene Zelle nicht behindert werden. Ein Kompetenzaspekt der Freiheit - ausgedrückt meist im Begriff der ,Autonomie“ - ist neben dem Hindernisaspekt immer vorhanden, und er ist, wie ich gleich zeigen will, der wichtigere.

Vielleicht lässt sich die vom Determinismus ausgehende Faszination durch die anhaltende Wirksamkeit der räumlichen Metaphorik erklären? Der Konflikt über diese Metaphorik (warum sollte sie auch nicht beibehalten werden?) kann auch als Konflikt über die sinnkritische Rolle der praktischen Handlungsinteressen im Rahmen des Problems der Willensfreiheit und der dieses Problem begleitenden Terminologie beschrieben werden. Geben unsere alläglichen Handlungsbedürfnisse den konstitutiven Rahmen ab, innerhalb dessen wir über

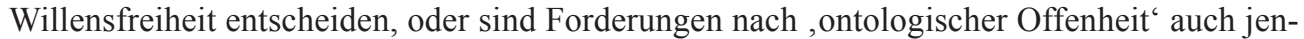
seits dieser Bedürfnisse sinnvoll? Seebaß ist sich dieser Alternative durchaus bewusst und folgt ihr sogar ein Stück in seiner Darstellung des Kompatibilismus (a, 147 f.), ohne freilich die ontologische Denkweise aufzugeben. Wie kann dieser Konflikt entschieden werden? Die einzig erfolgversprechende Strategie scheint darin zu bestehen, an unseren festen Intuitionen über Freiheit zu demonstrieren, dass sie durch den Determinismus nicht beunruhigt werden können. Die Festigkeit der Intuitionen muss aber gesucht werden, und sie findet sich meines Erachtens nur im Kontext praktischer Handlungsbedürfnisse.

Warum ist das bereits angedeutete Argument gegen den umfassenden Determinismus nicht hinreichend? Wenn alles, was wir tun, wie immer wir es tun, determiniert ist, so wäre das nur dann freiheitseinschränkend, wenn wir dazu Alternativen hätten. Wenn wir sie nicht haben, weil ein solcher Standpunkt für uns prinzipiell unzugänglich ist, ist er bedeutungslos. Vom 
Standpunkt möglichen Handelns her ist es deshalb irrelevant, ob ,alles' determiniert ist oder nicht. Alle Kriterien der inhaltlichen Freiheit könnten in einer vollkommen determinierten Welt erfüllt sein, sodass die real zugängliche Freiheit nicht berührt wird. Inkompatibilisten wie Seebaß scheinen von dieser Überlegung nicht beeindruckt, und es käme darauf an zu verstehen, warum.

Versuchen wir, die eben gemachte Überlegung in die Problematik der Wesensanalyse $\mathrm{zu}$ übersetzen. Angenommen, jemand ist, ohne es zu wissen, hypnotisiert, und aus seiner Sicht treffen alle Freiheitskriterien auf ihn zu. Angenommen, er habe keine Möglichkeit, die Hypnose zu entdecken, dann könnte er aus seiner Sicht sagen, er sei frei. Sofern es keine Möglichkeit für ihn gäbe, die Hypnose zu entdecken, wäre er, obwohl determiniert, auch frei. Ausgehend von einem solchen konkreten Beispiel könnte Seebaß eine Präzisierung des Möglichkeitsspielraums fordern, die zu diagnostizieren erlaubt, wann eine Determination im Allgemeinen eine Freiheitseinschränkung ist. Das schwer zu lösende Problem wird dann sichtbar, wenn wir statt von Hypnose von Erziehung reden und daran denken, welche psychologischen Dispositionen Eltern in Kindern festlegen. Unmöglich lässt sich ein präziser inhaltlicher Maßstab für die Möglichkeiten des Kindes finden, individuell oder allgemein, mit dem zwischen einschränkender und befreiender Erziehung unterschieden werden kann. Bleibt deshalb die Drohung der Unfreiheit bestehen?

Diese Frage und ihr Hintergrund sind insofern irreführend, als ihnen wiederum einseitig die Idee der Hindernisfreiheit zu Grunde liegt, die jede weitere Diskussion vor das Problem stellt, Freiheit im Gegensatz von Innen und Außen zu denken. Demgegenüber enthalten auch einfachste Situationen des Behindertwerdens durch Zäune oder Türen ein Wollen, von dessen Qualität es ebenfalls abhängt, inwieweit die Hindernisse tatsächlich freiheitseinschränkend sind. Die Fesseln eines psychiatrischen Patienten können insgesamt durchaus freiheitsfördernd sein, wenn sie zu seinem Gesunden beitragen. Unausweichlich ist im menschlichen Handeln immer ein Autonomie- oder Kompetenzaspekt enthalten, Freiheitstests nur nach dem Muster der Hindernisfreiheit anzulegen wird dem menschlichen Freiheitsphänomen nicht gerecht - anders als bei der Freiheit von Tieren, bei frei strömenden Flüssen oder einem sich vom Anker befreienden Schiff. Eine Lehre aus dieser Beobachtung ist, dass die Freiheit von Kindern eben nicht vorrangig durch indeterministische Einflüsse beschrieben werden sollte, sondern durch die freiheitskompetenten Eigenschaften, die auf Grund welcher Einflüsse auch immer entstehen.

Der Inkompatibilist verweigert sich typischerweise einer solchen Lehre und wirft die immer erneut ,offene Frage' (in der Tradition Moores) auf, ob die entsprechenden Eigenschaften nicht determiniert seien. Zwei mögliche Antworten auf diese offene Frage sind die praktische und die sinnkritische. Die praktische Antwort sagt, dass der Determinismus theoretisch gültig sein mag, aber seine Gültigkeit unser Leben nicht beeinträchtigt, also auch nicht Anlass für Fatalismus sein kann. Der Streit um Determinismus und Freiheit ist danach ein ,rein theoretischer' Streit, reserviert für Leute, die solche Herausforderungen lieben. Die sinnkritische Antwort sagt, dass der Gedanke der Freiheitseinschränkung in diesem Fall sinnlos ist und das Problem des Determinismus auf einer Verwirrung beruht.

Ich denke, dass diese zweite Kritik zu weit geht, dass aber der Nachteil für den Freiheitstheoretiker auf Grund der ersten Einschränkung bereits erheblich ist und es ausreicht, sich ihn klarer vor Augen zu führen. Illustrieren lässt sich das anhand des von Frankfurt erfundenen Szenarios, wonach einer Person unwissentlich die Alternative zu ihrer Entscheidung verschlossen ist, weil ein unbekannter Manipulator im alternativen Fall eingreifen würde. ${ }^{5}$

5 H. Frankfurt, Alternative Possibilities and Moral Responsibility, in: Journal of Philosophy, 66 (1969), 829-839. Ausführliche Diskussionen finden sich in: D. Widerker u. M. McKenna (Hg.), 
Frankfurt hat das Szenario auch deshalb eingeführt, um die Freiheitsfrage von der Problematik des Determinismus zu befreien. In unserem Kontext ist das Beispiel insofern interessant, als es die Frage aufwirft, ob der potenzielle Manipulator eine Freiheitseinschränkung darstellt oder nicht. Gemessen am Maßstab der Hindernisfreiheit ist die Antwort offensichtlich ,ja“. Aber warum sollte man diese Auffassung zu Grunde legen? Warum zählt nicht einfach, ob der Akteur nach den Autonomie-Kriterien seines Wollens frei ist? Für alle praktischen Belange, wie denen der moralischen Verantwortlichkeit und des authentischen Lebens, scheint das eindeutig der Fall zu sein. Moralisch ist der Akteur für sein Tun verantwortlich, und sein ernsthaftes Wollen ist im nicht-trivialen Fall fraglos Bestandteil seines Lebens.

Diejenigen, die nach wie vor die Drohung des Determinismus verspüren, müssten danach beachten, dass diese Drohung an das tatsächliche Leben, gemessen mit den Kriterien des tatsächlichen Lebens, nicht heranreicht. Warum sollte jedoch eine Drohung, die das nicht schafft, eine Drohung sein? Was wird von ihr bedroht? Offensichtlich nicht unser Handeln und Leben, sodass der Fatalist die üblichen Termini je anders verwenden muss, um sein Problem weiter zu formulieren. Das geschieht in Büchern wie denjenigen von Seebaß natürlich nicht, was zeigt, dass sie zu schreiben und zu lesen eindeutig vom praktischen Interesse an Freiheit motiviert ist. Würde dieses Interesse jedoch aufgegeben, und das sollte es um der Klarheit willen, kann Inkompatibilisten niemand daran hindern, ihren theoretischen Diskurs weiter zu führen. Beruhigend für uns praktisch Denkende ist jedoch, dass wir ihn getrost ignorieren können.

\section{Willensfreiheit, ein ,klassisches Problem'?}

Auf eine untergründige Weise ist in Seebaß' Texten die Neigung zur Ontologie mit einer Neigung zum Postulat, klassischer philosophischer Probleme', oder in Rortys Worten, zur doxographischen Auffassung von Philosophie verbunden, die ich in diesem letzten Abschnitt kritisieren werde. ${ }^{6}$ Seebaß' Diskussion der Willensfreiheit hat den kaum zu überschätzenden Vorzug, von einer ungewöhnlich umfangreichen und akribisch-historischen Dokumentation des Themas begleitet zu sein, die sich auf die meisten Klassiker und viele weniger bekannte Autoren der europäischen Philosophie und der jüdisch-christlichen Theologie erstreckt. Diese zusätzlichen Verweise und Kommentare sind durchweg informativ und ausgewogen, werfen aber allein ihres Umfangs wegen die Frage auf, welchen argumentativen Wert die Historiographie des Streits um Willensfreiheit haben soll. Seebaß selbst vertritt tendenziell die Position des Antihistorismus, also der historischen Universalität seines Themas. Gegenüber dem Verdacht der historischen Relativität des Willens- und Freiheitsdiskurses und reagierend vor allem auf Nietzsches Polemik gegen die Willensfreiheit als einer theologischen Erfindung mit Unterdrückungsfunktion, zeichnet er die Begriffsgeschichte der Willensfreiheit in der theologischen Theodizeeproblematik und in der altgriechisch-philosophischen Tradition nach (dies vor allem in b, Kap. 3).

Mir scheint, dass in diesem historischen Nachvollzug - gegen die Absicht des Autors eher Belege für eine historistische Sicht der Willensfreiheit zu Tage treten als für eine universelle. Historistisch liest man philosophische Probleme dann, wenn man sie so versteht, als

Moral Responsibility and Alternative Possibilities, Aldershot 2003; J. M. Fischer (Hg.), Free Will and Moral Responsibility, in: Midwest Studies in Philosophy, 29 (2005).

6 Vgl. auch R. Rorty, The Historiography of Philosophy: Four Genres, in: ders., Truth and Progress. Philosophical Papers, Bd. 3, Cambridge 1998. 
seien sie erst durch einen spezifischen kulturellen Rahmen als Probleme entstanden. Sicher sind die Phänomene des menschlichen Wollens (nicht, des ' Willens) und der Freiheit in allen Kulturen irgendwie präsent - ohne freilich an dieselbe Problematik gebunden sein zu müssen. In diesem Punkt ist Seebaß' Dokumentation eher irreführend, weil sie zu starr auf den plakativen genealogischen Einwand ausgerichtet und am Klassizismus philosophischer Probleme orientiert ist. Zu belegen vermag er sicher die Präsenz von Wollen und Wollensfreiheit in der griechischen Kultur vor dem Einfluss des Christentums sowie die steigende Bedeutung des Konflikts von Freiheit und Determinismus durch den Glauben an einen allmächtigen christlichen Gott, vor allem seit Augustinus. Die eigentliche historistische These, wonach die Art der philosophischen Probleme durch die kulturellen Rahmenbedingungen weitgehend vorentschieden ist, wird dadurch aber nicht widerlegt.

Angesichts des sichtbar gewordenen Gegensatzes von ,praktischen' und ,ontologischen Interessen' liegt die Vermutung nahe, dass einem ontologischen Freiheitsverständnis eher religiöse Motive zu Grunde liegen als einem praktischen, weil sich im ersten nicht-alltägliche Eigenschaften und Ziele ausdrücken, während das praktische Verständnis auf die Funktionen und Bedürfnisse des sozialen Zusammenlebens reagiert. Seebaß beachtet diesen Gegensatz in seiner historischen Rekonstruktion so wenig wie in seiner systematischen Diskussion. Durchweg geht es ihm um den Nachweis, dass die Idee der Willensfreiheit in Europa seit der griechisch-römischen Zeit präsent ist und durch die Kirchenväter nur radikalisiert und akzentuiert wurde (b, 128-130). Dieser Nachweis ist allerdings in Hinblick auf den Gegensatz von Willensfreiheit und Determinismus sowie auf die Frage, warum etwas ein philosophisches Problem wird, nicht sonderlich aussagekräftig.

So wird in Seebaß' Darstellung von Freiwilligkeit und akrasia bei Aristoteles deutlich, dass dieser „keine absolute, sondern nur eine relative Selbstbewegung im Auge hatte“ (b, 125), und dass er die „Freiwilligkeitsrückfrage an einem bestimmten Punkt abgebrochen“ $(\mathrm{b}, 125)$ hat. Aristoteles interessierte sich eben nicht sonderlich für die praktischen Konsequenzen aus dem in De Interpretatione erörterten Determinismus. Er sah keine allgemeine Bedrohung eines ,freien Willens', sondern war in der Ethik vorrangig an der Willensschwäche, wenn auch an einigen Stellen am psychologischen Determinismus interessiert. ${ }^{7}$ An eine universelle Kausaltheorie oder einen das Theodizee-Problem aufwerfenden Gott musste er nicht denken, sodass es mindestens irreführend ist, wenn Seebaß bei Aristoteles, wie auch bei Platon und anderen, freies Wollen mit „Indeterminismus“ assoziiert (b, 123-126) und damit eine gleich bleibende Bedeutung des Problems unterstellt. Welchen Nachweis der Universalität hat man gewonnen, wenn man bereits den blinden Zufall als Indeterminismus bezeichnet? Offenkundig entstand der Gegensatz von Willensfreiheit und Determinismus erst mit Lukrez, und das unter Zuhilfenahme der demokritschen Atomtheorie, worin sich eine bis zur heutigen Neurodebatte wirksame Motivation dieses Konflikts durch die Naturwissenschaften anmeldet.

Während das philosophische Interesse an Willensfreiheit in der theologischen Tradition, wie Seebaß gut dokumentiert (b, 90-116), aus der Theodizee-Debatte und den Schwierigkeiten entspringt, dem freien Willen in einer von Gott determinierten Welt einen Sinn zu

7 Siehe die etwas unterschiedliche Darstellung bei: P. Huby, The First Discovery of the Freewill Problem, in: Philosophy, 42 (1967), 353-362; und W. F. R. Hardie, Aristotle and the Freewill Problem, in: Philosophy, 43 (1968), 274-278. „If we define the freewill problem as arising only for a philosopher who starts by accepting, or finding undeniable, a deterministic metaphysical doctrine, then Aristotle, let us allow, did not discover the problem. But he anticipated some of the moves which have been made by modern philosophers in their discussion of the problem." (Ebd., 278) 
geben, entzündet es sich in der unmittelbaren Gegenwart an der naturalistischen Provokation durch die Neurowissenschaften, der Seebaß keine vergleichbare Aufmerksamkeit schenkt (a, 201-206). Aus diesen historischen Kontexten heraus entspringt ein je verschiedenes Interesse am philosophischen Begriff der Willensfreiheit, in der Gegenwart verbunden mit der Rolle und der Bedeutung naturalistischer Theorien. Demgegenüber schlägt Seebaß vor, dass allein die allgemeine Fragemöglichkeit nach Begriffen wie dem der Willensfreiheit bereits ein abstraktes, diesen Kontexten entzogenes Interesse erzeugen sollte. Ihm zufolge können Fragen normativer sozialer Kontrolle wie andere ,speziellere Fragen nach der Indeterminiertheit des Willens“ (b, 25) die ,generelle Frage der Willensfreiheit" nicht erschöpfen. Seebaß möchte so die ,generelle Frage“ von konkreten und benennbaren Unfreiheiten abgekoppelt und zu einer freistehenden Reflexionsquelle erhoben sehen. In seinen beiden noch ausstehenden Bänden muss er uns allerdings erst noch überzeugen, welchen Verlust wir erleiden, wenn wir dieses ontologische Bedürfnis nicht teilen. 